\title{
Symbolverzeichnis Band VI
}

\author{
(alphabetisch)
}

\begin{tabular}{|c|c|}
\hline$A$ & Richardson-Konstante \\
\hline$a$ & Potenzialstärke (Born-Mayer-Potenzial), Gitterkonstante \\
\hline$\vec{a}, \vec{b}, \vec{c}$ & fundamentale Translationsvektoren des direkten Gitters \\
\hline$\vec{a}^{\star}, \vec{b}^{\star}, \vec{c}^{\star}$ & Basisvektoren des reziproken Gitters \\
\hline$A_{H}$ & Hall-Koeffizient \\
\hline AS & Antistrukturatom \\
\hline$A_{s}, A_{f}$ & $\begin{array}{l}\text { Start- und Endtemperatur der Rückbildung der Martensitphase in die Ausgangs- } \\
\text { phase }\end{array}$ \\
\hline$B$ & „Magnetfeld“ (magnetische Kraftflussdichte) \\
\hline$b$ & Reichweite (Born-Mayer-Potenzial) \\
\hline$\vec{b}$ & Burgers-Vektor \\
\hline$B Z$ & Brillouin-Zone \\
\hline$c$ & Vakuumlichtgeschwindigkeit ( $c=299792458$ m/s, exakt) \\
\hline$\vec{C}_{h}$ & Vektor der Rollrichtung (Nanoröhrchen, $\vec{C}_{h}=n \cdot \vec{a}_{1}+m \cdot \vec{a}_{2}$ ) \\
\hline$C_{V}$ & Wärmekapazität bei konstantem Volumen \\
\hline$c_{V}$ & spezifische Wärme bei konstantem Volumen \\
\hline$C_{V}^{(m)}$ & molare Wärmekapazität (Molwärme) \\
\hline$d$ & Netzebenenabstand \\
\hline DLS & Doppelleerstelle \\
\hline$d_{s}^{p}, d_{l}^{p}, d_{s}^{o}, d_{l}^{o}$ & $\begin{array}{l}\text { kurze und lange Diagonale des prolaten und des oblaten Rhomboeders } \\
\text { (Strukturelemente von Quasikristallen) }\end{array}$ \\
\hline$e$ & $\begin{array}{l}\text { Elementarladung ( } e=1,602 \cdot 10^{-19} \mathrm{C} \text {; genauer Wert: } e=1,602176634 \cdot 10^{-19} \mathrm{C} \text {, } \\
\text { exakt) }\end{array}$ \\
\hline$\vec{E}$ & elektrische Feldstärke \\
\hline$e^{-}$ & Elektron \\
\hline$E, E_{\mathrm{kin}}, E_{\mathrm{pot}}$ & Energie, kinetische und potenzielle Energie \\
\hline ECAP & equal channel angular pressing \\
\hline$E_{k}$ & Kontaktpotenzial \\
\hline$E_{n}$ & Neutronenenergie \\
\hline$\vec{e}_{n}$ & Normaleneinheitsvektor \\
\hline$E_{S}$ & $\begin{array}{l}\text { Energie der Gitterschwingung (Phonon, } E_{S}=\hbar \Omega \text { ), Tiefe des Potenzialtopfes } \\
\text { (Austrittsarbeit) }\end{array}$ \\
\hline$F$ & freie Energie $(F=U-T S)$, Kraft \\
\hline$f$ & Kraftkonstante (Federkonstante) \\
\hline$f^{(1)}(\vec{r}), f^{(2)}\left(\vec{r}_{1}, \vec{r}_{2}\right)$ & Einteilchen- und Zweiteilchen-Dichtefunktion \\
\hline$f(\theta)$ & Atomformfaktor (Atom-Streuamplitude) \\
\hline$F A$ & Fremdatom \\
\hline$f_{E}, f_{D}$ & Einstein-Funktion, Debye-Funktion \\
\hline FGL & Formgedächtnis-Legierung (shape memory alloy, SMA) \\
\hline$F(\overrightarrow{\Delta k})$ & Strukturamplitude (auch Strukturfaktor genannt) \\
\hline G & Schubmodul \\
\hline GG & Gleichgewicht, Gleichgewichtszustand \\
\hline$\vec{G}$ & Gittervektor des reziproken Gitters $\left(\vec{G}=h \vec{a}^{\star}+k \vec{b}^{\star}+l \vec{c}^{\star}\right)$ \\
\hline$g(r)$ & Paarverteilungsfunktion \\
\hline$g\left(\vec{r}_{1}, \vec{r}_{2}\right)$ & Zweiteilchen-Verteilungsfunktion \\
\hline$H$ & Enthalpie \\
\hline
\end{tabular}


$h(r) \quad$ Paarkorrelationsfunktion $(h(r)=g(r)-1)$

$h, h \quad$ Plancksches Wirkungsquantum $\left(h=6,6261 \cdot 10^{-34} \mathrm{Js}\right.$;

genauer Wert: $h=6,62607015 \cdot 10^{-34} \mathrm{Js}$, exakt),

reduziertes Plancksches Wirkungsquantum $\hbar=\frac{h}{2 \pi}$

$h_{0}$

Zellvolumen des klassischen Phasenraumes $\left(h_{0}=\delta q \cdot \delta p\right)$

$(h k l),[h k l] \quad$ Miller Indizes einer Kristallebene (Ebenenschar) und einer Kristallrichtung

hdp hexagonal dichtest gepack

$h_{j}$

HPTS

ITO

$j_{E m}$

relative Häufigkeit für Ergebnis $j$

high pressure torsion straining

durchsichtige Elektrode aus Indium-Zinn Oxid

Emissionsstromdichte

$j_{\mathrm{x}}$

Stromdichte in $x$-Richtung

Boltzmannkonstante, Direktionskraft (Pendel)

Kompressionsmodul

Radius der Fermi-Kugel

kubisch-flächenzentriert

Korngrenze

kubisch-raumzentriert

Lorenz-Zahl (Gesetz von Wiedemann und Franz)

Distanz eines Einzelsprungs (Zufallsbewegung),

Drehimpulsquantenzahl

LCSLM

liquid crystal spatial light modulators

$L_{v, S}(v, T)$

$L S$

$M$

spektrale Strahldichte des schwarzen Körpers

(Gitter-)Leerstelle

Anzahl einander ausschließender Ergebnisse, Magnetisierung, magnetisches Gesamtmoment

$m$

$M_{A}$

$m_{e}^{\star}$

resultierende Verschiebung (Zufallsbewegung, $m=n_{1}-n_{2}$ )

Molmasse $\left(M_{A}=\frac{m}{v}\right)$

effektive Masse der Leitungselektronen

magnetische Spinquantenzahl

Start- und Endtemperatur der Ausbildung der Martensitphase

$M_{s}, M_{f}$

$M W N T$

multi-walled nanotube

$N$

Teilchenzahl

$N, N_{j}$

$n$

$\vec{n}$

$N_{A}$

$\vec{n}(\vec{r})$

$n_{1}, n_{2}$

$N G G$

Gesamtzahl der Messungen, Anzahl der Messungen mit Ergebnis $j$

Hauptquantenzahl (Energiequantenzahl), Ordnung der Beugung, Brechzahl

Normalvektor

Avogadrozahl

Direktorfeld (achsialer Vektor, Flüssigkristalle)

Anzahl der Verschiebungen nach rechts oder links

(eindimensionale Zufallsbewegung)

$n_{i}$

Nichtgleichgewichtszustand

$n_{Q}(T)$

Quantenzahlen

Quantenkonzentration

$n_{r}^{S_{z}}$

$\bar{n}_{s}$

$P$

Anzahl der Teilchen im Zustand $r$ und $S_{z}$

mittlere Besetzungszahl des Zustands $s$ mit Energie $\varepsilon_{s}$

Druck

$P(x) d x \quad$ Standardform der Gaußverteilung für die kontinuierliche Variable $x$

$P^{*}$

normierte Wahrscheinlichkeit 


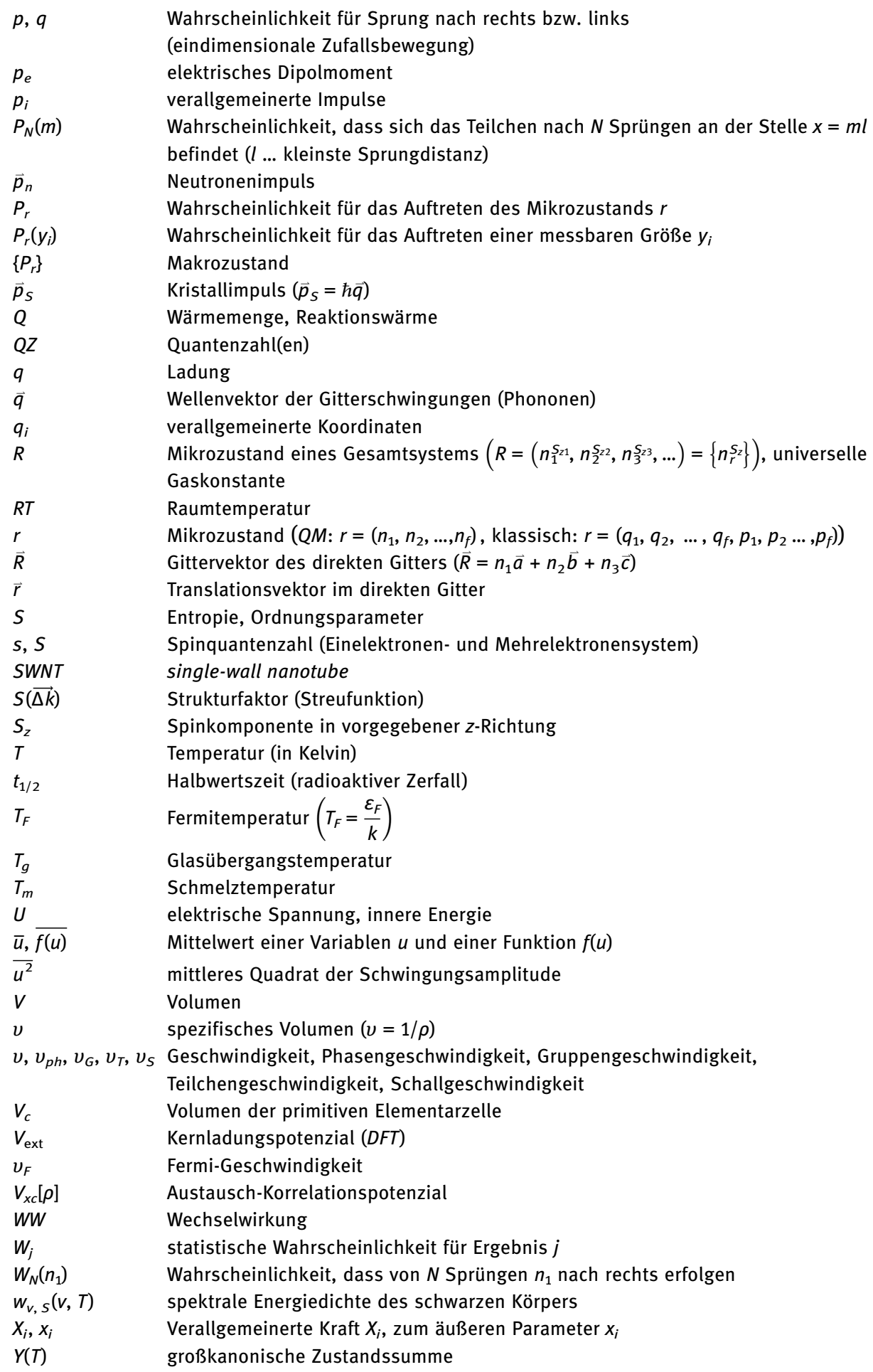


$Z(E), z(E)$

$Z(T)$

$z(\omega)$

ZGA

$Z_{1}$

$Z_{\Omega}(\Omega)$

$Z_{\omega}(\omega), z_{\omega}(\omega)$

$\alpha$

$\beta=\frac{1}{k T}$

$\Gamma$

$\overrightarrow{\Delta k}$

$\overline{(\Delta u)^{2}}$

$\Delta^{*} u$

$\Delta x_{h}$

$\delta_{i j}$

$\varepsilon$

$\varepsilon_{0}$

$\varepsilon_{F}$

$\varepsilon_{r}$

$\eta$

$\theta$

$\theta_{E}, \theta_{D}$

$\vartheta$

$\vartheta_{T}$

$\Lambda$

$\lambda$

$\lambda_{F}$

$\lambda_{\text {StoB }}$

$\lambda_{T}$

$\lambda_{z}$

$\mu$

$v$

$\rho$

$\rho_{0}$

$\rho(E)$

$\sigma$

$\tau$

$\Phi$

$\Phi(x)$

$\varphi_{i}$

$\psi(\vec{r})$

$\psi_{+}, \psi$.
Zahl der Zustände (der Wellenfunktionen), Zustandsdichte

kanonische Zustandssumme

Modendichte

Zwischengitteratom

Einteilchen-Zustandssumme

spektrale Modenzahl der Phononen

spektrale Modenzahl, spektrale Modendichte

Polarisierbarkeit, Madelung-Konstante $\left(\alpha=\sum_{j}( \pm) \frac{1}{p_{i j}}\right)$, linearer
Ausdehnungskoeffizient

thermodynamischer Parameter

Gibbsscher Phasenraum (Г-Raum, Zustandsraum)

Streuvektor $\left(\overrightarrow{\Delta k}=\vec{k}^{\prime}-\vec{k}\right)$

Schwankungsquadrat (Varianz) von $u$ (bei der Zufallbewegung: $\overline{\left(\Delta n_{1}\right)^{2}}$

Standardabweichung (mittlere quadratische Abweichung: $\Delta^{*} u=+\sqrt{\overline{(\Delta u)^{2}}}$, bei der Zufallsbewegung: $\Delta^{\star} n_{1}=\sqrt{\overline{\left(\Delta n_{1}\right)^{2}}}$

Halbwertsbreite der Gaußverteilung

Kronecker Symbol $\left(\delta_{i j}=1\right.$ für $i=j$ und $\delta_{i j}=0$ für $\left.i \neq j\right)$

Potenzialtiefe (Lennard-Jones-Potenzial)

elektrische Feldkonstante (Influenzkonstante)

Fermienergie $\left(\varepsilon_{F}=\mu(T=0)\right)$

Energie eines Teilchens im Zustand $r$ ( und $S_{z}$ )

Viskosität

Beugungswinkel (Glanzwinkel bei der Röntgenbeugung)

Einstein-Temperatur, Debye-Temperatur

Temperatur in ${ }^{\circ} \mathrm{C}$

Debye-Waller-Faktor (Temperaturfaktor der Röntgenbeugung)

Wärmeleitfähigkeit

Mittelwert bei der Poissonverteilung $\left(\lambda=\bar{n}_{1}\right)$, Wellenlänge

Fermi-Wellenlänge

mittlere freie Weglänge

thermische de Broglie-Wellenlänge

Zerfallskonstante (radioaktiver Zerfall)

magnetisches Moment eines Teilchens, chemisches Potenzial

Frequenz, Stoffmenge (Molzahl)

spezifischer elektrischer Widerstand $\left(\sigma=\frac{1}{\rho}\right)$

mittlere Teilchendichte

Zustandsdichte

Standardabweichung $\left(\sigma=\sqrt{\overline{(\Delta x)^{2}}}=I \sqrt{\overline{(\Delta m)^{2}}}\right)$, Kontaktabstand (Lennard-Jones-

Potenzial), elektrische Leitfähigkeit

Schubspannung (Scherspannung), Stoßzeit (Relaxationszeit)

Austrittsarbeit

Debye-Funktion

Kohn-Sham-Orbitale

Wellenfunktion (stationäres Problem)

symmetrische und antisymmetrische Wellenfunktion 


$\begin{array}{ll}\omega & \text { Kreisfrequenz }(\omega=2 \pi v) \\ \Omega(E) & \text { mikrokanonische Zustandssumme } \\ \Omega(\bar{q}) & \text { Kreisfrequenz der Gitterschwingungen (Phononen) } \\ \Omega_{+}, \Omega_{-} & \text {Frequenz der optischen und der akustischen Phononen } \\ \omega_{E}, \omega_{D} & \text { Einstein-Frequenz, Debye-Frequenz } \\ \Omega_{k} & \text { Volumen eines Zustands in der Fermi-Kugel }\end{array}$

\section{Wichtige physikalische Größen, Band VI}

Universelle Gaskonstante (molar gas constant) Boltzmannkonstante Avogadro-Zahl Plancksches Wirkungsquantum reduziertes Plancksches Wirkungsquantum

Elementarladung Lichtgeschwindigkeit Masse des Elektrons

atomare Masseneinheit (amu)

Energieumrechnung

Influenzkonstante (electric permittivity)

$$
\begin{aligned}
& R=k \cdot N_{A}=8,314462618 \ldots \mathrm{J} \cdot \mathrm{mol}^{-1} \cdot \mathrm{K}^{-1} \text {, exakt } \\
& k=1,380649 \cdot 10^{-23} \mathrm{~J} \cdot \mathrm{K}^{-1}=8,617333262 \ldots \cdot 10^{-5} \mathrm{eVK}^{-1} \text {, exakt } \\
& N_{A}=6,02214076 \cdot 10^{23} \mathrm{~mol}^{-1} \text {, exakt } \\
& h=6,62607015 \cdot 10^{-34} \mathrm{Js}=4,135667696 \ldots \cdot 10^{-15} \mathrm{eV} \mathrm{s} \text {, exakt } \\
& \hbar=\frac{h}{2 \pi}=1,054571817 \ldots \cdot 10^{-34} \mathrm{Js}=6,582119569 \ldots \cdot 10^{-16} \mathrm{eV} \mathrm{s}, \\
& \text { exakt } \\
& e=(1,602176634 \pm 0,0000000098) \cdot 10^{-19} \mathrm{C}, \text { exakt } \\
& c=299792458 \mathrm{~m} / \mathrm{s} \text {, exakt } \\
& m_{e}=(9,1093837015 \pm 0,0000000028) \cdot 10^{-31} \mathrm{~kg}= \\
& =(0,51099895000 \pm 0,00000000015) \mathrm{MeV} / \mathrm{c}^{2}= \\
& =(5,48579909065 \pm 0,00000000016) \cdot 10^{-4} u \\
& 1 \mathrm{u}=(1,66053906660 \pm 0,00000000050) \cdot 10^{-27} \mathrm{~kg}= \\
& =(931,49410242 \pm 0,0000000028) \mathrm{MeV} / \mathrm{c}^{2} \\
& 1 \mathrm{eV}=1,602176634 \cdot 10^{-19} \mathrm{~J} \text {, exakt } \\
& 1 \mathrm{~J}=(6,241509074 \ldots \pm 0,0000000382) \cdot 10^{18} \mathrm{eV} \\
& \varepsilon_{0}=(8,8541878128 \pm 0,0000000013) \cdot 10^{-12} \mathrm{Fm}^{-1}\left(\text { oder } \mathrm{AsV}^{-1} \mathrm{~m}^{-1}\right. \\
& \text { oder } \mathrm{C}^{2} \mathrm{~N}^{-1} \mathrm{~m}^{-2} \text { ) } \\
& \frac{1}{4 \pi \varepsilon_{0}}=8,98755 \cdot 10^{9} \mathrm{Nm}^{2} / \mathrm{C}^{2}
\end{aligned}
$$


